\title{
Implantable Cardioverter-Defibrillators and Patient-Reported Outcomes in Adults with Congenital Heart Disease: an International Study
}

Valérie Lévesque, MD; ${ }^{1}$ Laurence Laplante, $\mathrm{MD} ;{ }^{1}$ Azadeh Shohoudi, $\mathrm{PhD}{ }^{1}$; Silke Apers, $\mathrm{PhD}{ }^{2}$ Adrienne H. Kovacs, $\mathrm{PhD} ;{ }^{3}$ Koen Luyckx, $\mathrm{PhD} ;{ }^{4}$ Corina Thomet, $\mathrm{MS} ;{ }^{5}$ Werner Budts, MD, PhD; ${ }^{6}$ Junko Enomoto, PhD; $;^{7}$ Maayke A. Sluman, MD, PhD $;{ }^{8}$ Chun-Wei Lu, PhD $;{ }^{9}$ Jamie L. Jackson, PhD $;{ }^{10}$ Stephen C. Cook, MD $;{ }^{11}$ Shanthi Chidambarathanu, MD $;{ }^{12}$ Luis Alday, MD $;{ }^{13}$ Katrine Eriksen, MSc; ${ }^{14}$ Mikael Dellborg, MD, PhD; ${ }^{15}$ Malin Berghammer, PhD; ${ }^{16}$ Bengt Johansson, MD, PhD; ${ }^{17}$

Andrew S Mackie, MD, PhD; ${ }^{18}$ Samuel Menahem, MD $;{ }^{19}$ Maryanne Caruana, MD, PhD $;{ }^{20}$

Gruschen Veldtman, MD; ${ }^{21}$ Alexandra Soufi, MD; ${ }^{22}$ Susan M. Fernandes, LPD, PA-C; ${ }^{23}$

Kamila White, PhD $;^{24}$ Edward Callus, $\mathrm{PhD} ;{ }^{25}$ Shelby Kutty, MD, PhD ${ }^{26}$ Judith Brouillette, MD, PhD ${ }^{1}$

Benjamin Casteigt, $\mathrm{MD} ;{ }^{1}$ Philip Moons, $\mathrm{PhD} ;{ }^{27}$ Paul Khairy, $\mathrm{MD}, \mathrm{PhD}^{1}$; on behalf of the APPROACH-IS consortium and the International Society for Adult Congenital Heart Disease (ISACHD)

${ }^{1}$ Montreal Heart Institute, Université de Montréal, Montreal, Canada

${ }^{2}$ KU Leuven Department of Public Health and Primary Care, University of Leuven, Leuven, Belgium

${ }^{3}$ Knight Cardiovascular Institute, Oregon Health \& Science University, Portland, Oregon

${ }^{4}$ KU Leuven - University of Leuven, Psychology and Development in Context, B-3000 Leuven, Belgium; UNIBS, University of the Free State, Bloemfontein, South Africa

${ }^{5}$ Center for Congenital Heart Disease, Inselspital-Bern University Hospital, University of Bern, Bern, Switzerland

${ }^{6}$ Division of Congenital and Structural Cardiology, University Hospitals Leuven and Department of Cardiovascular Sciences, KU Leuven, Leuven, Belgium

${ }^{7}$ Department of Adult Congenital Heart Disease, Chiba Cardiovascular Center, Chiba, Japan

${ }^{8}$ Department of Cardiology, Jeroen Bosch Hospital, Hertogenbosch, the Netherlands and Coronel Institute for occupational health, Academic Medical Centre, Amsterdam, the Netherlands

${ }^{9}$ Department of Pediatrics, National Taiwan University Hospital, Taipei, Taiwan

${ }^{10}$ Center for Biobehavioral Health, Nationwide Children's Hospital, Columbus, Ohio

${ }^{11}$ Adult Congenital Heart Disease Center, Helen DeVos Children's Hospital, Grand Rapids, Michigan

${ }^{12}$ Frontier Lifeline Hospital (Dr. K.M. Cherian Heart Foundation), Chennai, India

${ }^{13}$ Division of Cardiology, Hospital de Niños, Córdoba, Argentina

${ }^{14}$ Department of Cardiology, Oslo University Hospital—Rikshospitalet, Oslo, Norway

${ }^{15}$ Adult Congenital Heart Unit, Sahlgrenska University Hospital/Östra, Gothenburg, Sweden

${ }^{16}$ Centre for Person-Centred Care (GPCC), University of Gothenburg and Department of Health Sciences, University West, Trollhättan, Sweden

${ }^{17}$ Department of Public Health and Clinical Medicine, Umeå University, Umeå, Sweden

${ }^{18}$ Division of Cardiology, Stollery Children's Hospital, University of Alberta, Edmonton, Canada

${ }^{19}$ Monash Medical Centre, Monash University, Melbourne, Australia

${ }^{20}$ Department of Cardiology, Mater Dei Hospital, Birkirkara Bypass, Malta

${ }^{21}$ Adult Congenital Heart Disease Center, Cincinnati Children's Hospital, Cincinnati, Ohio

${ }^{22}$ Department of Congenital Heart Disease, Louis Pradel Hospital, Lyon, France

${ }^{23}$ Department of Pediatrics and Medicine, Stanford University School of Medicine, Palo Alto, California

${ }^{24}$ Adult Congenital Heart Disease Center, Washington University and Barnes Jewish Heart \& Vascular Center, University of Missouri, Saint Louis, Missouri

${ }^{25}$ Clinical Psychology Service, IRCCS Policlinico San Donato, Milan, Italy and Department of Biomedical Sciences for Health, Università degli Studi di Milano, Milan, Italy

${ }^{26}$ Adult Congenital Heart Disease Center, University of Nebraska Medical Center/Children's Hospital \& Medical Center, Omaha, Nebraska

${ }^{27}$ KU Leuven-University of Leuven, Leuven, Belgium; Institute of Health and Care Sciences, University of Gothenburg, Gothenburg, Sweden; Department of Paediatrics and Child Health, University of Cape Town, Cape Town, South Africa

Short title: ICDs and patient-reported outcomes in ACHD

Word count: 5,000

Funding sources: This work was supported by the Research Fund - KU Leuven (Leuven, Belgium) grant OT/11/033; Swedish Heart-Lung Foundation grant 20130607; University of Gothenburg Centre for Person-centred Care; and Cardiac Children's Foundation (Taiwan) grant CCF2013_02.

Conflict of interest statement: No conflicts of interest to disclose.

Correspondence: Dr. Paul Khairy, Montreal Heart Institute, 5000 Belanger St, Montreal, Quebec, Canada, H1T 1C8; E-mail: paul.khairy@umontreal.ca 


\begin{abstract}
Background-Implantable cardioverter-defibrillators (ICDs) are increasingly used to prevent sudden deaths in the growing population of adults with congenital heart disease (CHD). Yet, little is known about their impact on patient-reported outcomes (PROs).

Objective-We assessed and compared PROs in adults with CHD with and without ICDs.

Methods-A propensity-based matching weight analysis was conducted to evaluate PROs in an international cross-sectional study of adults with CHD from 15 countries across 5 continents.
\end{abstract}

Results-A total of 3,188 patients were included: 107 with ICDs and 3,081 weight-matched controls without ICDs. ICD recipients averaged $40.1 \pm 12.4$ years of age, with $>95 \%$ having moderate or complex CHD. Defibrillators were implanted for primary and secondary prevention in $38.3 \%$ and $61.7 \%$, respectively. Perceived health status, psychological distress, sense of coherence, and health behaviours did not differ significantly in patients with and without ICDs. However, ICD recipients had a more threatening view of their illness (relative \% difference $8.56, \mathrm{P}=0.011$ ). Those with secondary compared to primary prevention indications had a significantly lower quality of life score (linear analogue scale $72.0 \pm 23.1$ versus $79.2 \pm 13.0, \mathrm{P}=0.047$ ). Marked geographic variations were observed. Overall sense of well-being, assessed by a summary score that combines various PROs, was significantly lower in ICD recipients (versus controls) from Switzerland, Argentina, Taiwan, and USA.

Conclusions - In an international cohort of adults with CHD, ICDs were associated with a more threatening illness perception, with a lower quality of life in those with secondary compared to primary prevention indications. However, marked geographic variability in PROs was observed.

Key words: adult congenital heart disease; implantable cardioverter-defibrillator; quality of life; patient-reported outcomes; sudden cardiac death 


\section{INTRODUCTION}

Sudden cardiac death is a leading cause of mortality in adults with congenital heart disease (CHD) such that defibrillators are increasingly implanted in this growing population. ${ }^{1}$ While most patients appear to adjust well to this therapy, considerable psychosocial issues have been reported in the general population with implantable cardioverter-defibrillators (ICDs). ${ }^{2}$ Nevertheless, there is a paucity of data that specifically addresses the impact of ICDs on quality of life and other patient-reported outcomes (PROs) in adults with CHD. Understanding the influence of ICDs on PROs is of particular importance considering the high baseline prevalence of psychosocial concerns in adults with CHD, including generalized anxiety, depression, and difficulties coping with a life-long medical condition. ${ }^{3}$ Indeed, targeted therapy to reduce psychological distress has been recognized as an important component of comprehensive care for adults with CHD. ${ }^{4}$

We, therefore, sought to assess the impact of ICDs in the largest study of PROs in adults with CHD, i.e., Assessment of Patterns of Patient-Reported Outcomes in Adults with Congenital Heart disease - International Study (APPROACH-IS), which enrolled over 4000 patients from 15 countries across 5 continents. ${ }^{5}$ Outcomes of interest included quality of life, perceived health status, psychological distress, sense of coherence, illness perception, and health behaviours.

\section{METHODS}

\section{Patient selection}

Design and methods of APPROACH-IS have previously been described (ClinicalTrials.gov NCT02150603). ${ }^{5}$ In short, the study included adults ( $\geq 18$ years of age) diagnosed with CHD before 10 years of age with the physical, cognitive, and language capabilities required to complete self-reported questionnaires. A total of 4028 patients from 24 centers were recruited from the following countries via the International Society for Adult Congenital Heart Disease 
(ISACHD): Argentina, Australia, Belgium, Canada, France, India, Italy, Japan, Malta, Norway, Taiwan, the Netherlands, Sweden, Switzerland and USA. All patients enrolled in APPROACH-IS with complete data regarding ICD history and PROs were retained. In order to assess geographic variations, participating countries were analyzed individually and divided into the following four regions: Americas (Canada, USA, and Argentina), Europe (Belgium, France, Italy, Malta, Norway, Sweden, Switzerland and the Netherlands), Eastern (Taiwan and India), and Pacific (Australia and Japan).

\section{Data collection and ICDs}

Demographic data including age, sex, ethnicity, educational level, and marital status were collected using a self-reported questionnaire. Type and complexity of CHD, history of congestive heart failure, and details regarding ICDs were extracted from medical records by site investigators. Complexity of CHD was categorized as simple, moderate, or complex according to a previously defined classification scheme. ${ }^{6}$ The presence or absence of an ICD was noted, along with date of surgery and whether the device was implanted for primary or secondary prevention against sudden cardiac death. All subjects provided written informed consent to participate. The study was approved by the local institutional review board of each participating center and was conducted in accordance with the International Council of Harmonization Tripartite Guidelines for Good Clinical Practice.

\section{Patient-reported outcomes}

A series of questionnaires were administered in the patient's language to assess the following PROs summarized in Table 1: quality of life, perceived health status, psychological distress, sense of coherence, illness perception, and health behaviours. Quality of life, conceptually 
defined as the degree of overall satisfaction with life, ${ }^{7}$ was assessed by means of a linear analogue scale (LAS) and the Satisfaction With Life Scale (SWLS). The LAS consists of a vertical graded line that ranges from worst (0) to best (100) imaginable quality of life. The SWLS is a 5-question survey that assesses the individual's global judgement of life satisfaction. ${ }^{8}$ Perceived health status was defined as the patient's perception of the impact of the disease on symptoms, functional status, and health-related quality of life. ${ }^{9}$ It was assessed by the 12 -item Short-Form Health Survey version 2 (SF-12), which is divided into physical (PCS) and mental (MCS) component scores, along with the European Quality of Life-5 Dimension (EQ-5D) score. ${ }^{10}$ Two categories of psychological distress were assessed, i.e., anxiety and depression, by means of the Hospital Anxiety (HADS-Anxiety) and Depression (HADS-Depression) Scale. ${ }^{11}$ Sense of coherence refers to the individual's generalized view that stimuli are structured and predictable, resources are available to meet associated demands, and that addressing these demands is worthy of investment. ${ }^{12}$ It was evaluated using the 13 -item Orientation to Life Questionnaire (SOC).$^{13}$ Illness perception, defined as cognitive representations and beliefs about one's illness, was assessed using the Brief Illness Perception Questionnaire (Brief IPQ). ${ }^{14}$ The Health-Behaviour Scale-Congenital Heart Disease (HBS-CHD) questionnaire targets behaviours related to substance abuse, dental hygiene, and physical activity. ${ }^{15}$ Finally, a composite score developed by APPROACH-IS investigators (APPRtot) was used as a summary measure to capture the various PROs. ${ }^{16}$ A higher composite score indicates a superior state of well-being.

\section{Statistical analysis}

Continuous variables are summarized as mean \pm standard deviation and categorical variables by frequency and percentage. The impact of ICDs on PROs was assessed using propensity-matched analyses. Propensity scores were estimated from a non-parsimonious multivariable logistic 
regression model in which ICD therapy was modelled as the dependent variable and patient-level variables listed in Table 2 were included as covariates. Covariates were limited to variables with a low proportion of missing values $(<5 \%)$.

A matching weight (MW) approach was used, which is an extension of inverse probability of treatment weighting that reweights both treatment groups to render them as comparable as possible. ${ }^{17}$ In Figure 1, a LOVE plot depicts the percentage of pooled weighted standard deviations for the values of standard differences in weighted means or weighted proportions for each covariate across exposure groups. After applying MWs, an absolute standard difference $<10 \%$ was obtained for all covariates, indicating excellent balance between groups. To assess regional variations, differences in weighted means of PROs among the two exposure groups were assessed according to four geographical regions. Two-sided P-values $<0.05$ were considered statistically significant. No adjustments were made for multiple comparisons considering the exploratory nature of the study. All analyses were performed using SAS software version 9.4 (SAS Institute, Cary, North Carolina) and R version 3.2.5.

\section{RESULTS}

\section{Study population}

A total of 3,188 patients met inclusion criteria for the APPROACH-IS ICD study: 107 with ICDs and 3,081 weight-matched controls without ICDs. Patients with ICDs were distributed according to the following countries of domicile: Argentina $\mathrm{N}=3$, Australia $\mathrm{N}=2$, Belgium $\mathrm{N}=10$, Canada $\mathrm{N}=16$, Italy $\mathrm{N}=1$, Japan $\mathrm{N}=3$, Norway $\mathrm{N}=12$, Sweden $\mathrm{N}=1$, Switzerland $\mathrm{N}=6$, Taiwan $\mathrm{N}=3$, the Netherlands $\mathrm{N}=10$, and USA $\mathrm{N}=40$. Characteristics of the two matched groups are summarized in Table 2. No statistically significant difference was observed for any characteristic. The average age of patients with and without ICDs was $40.1 \pm 12.4$ versus $40.2 \pm 14.1$ years $(\mathrm{P}=0.462)$. Overall, 
$50.5 \%$ and $50.4 \%$ of the matched cohorts with and without ICDs were female $(\mathrm{P}=0.985)$. In both groups, over $95 \%$ of patients had moderate or complex CHD.

From the 107 patients with ICDs, 41 (38.3\%) were implanted for primary and $66(61.7 \%)$

for secondary prevention. Applying the MW approach to the ICD population, 104 of 107 (97.2\%) patients were retained for predefined secondary analyses comparing PROs in those with primary versus secondary prevention indications. As shown in Table 3, there were no significant differences in characteristics between matched cohorts. On average, ICDs were implanted $7.3 \pm 8.1$ versus $7.6 \pm 7.1$ years prior to the assessment of PROs in patients with primary versus secondary prevention indications $(\mathrm{P}=0.852)$.

\section{Impact of ICDs on PROs}

Comparisons of PROs in matched cohorts with and without an ICD and in those with primary versus secondary prevention indications are presented in Table 4. No differences in quality of life, perceived health status, psychological distress, sense of coherence, health behaviours, or composite summary score were noted in adults with CHD with and without ICDs. However, patients with ICDs had a significantly more threatening view of their illness (Brief IPQ score $37.7 \pm 12.1$ versus $34.7 \pm 13.8$, relative $\%$ difference $8.56, \mathrm{P}=0.011$ ). Moreover, ICD recipients exhibited a non-significant trend towards having a worse perceived physical health status score (SF-12 PCS 66.5 \pm 22.6 versus $70.2 \pm 23.3$, relative $\%$ difference $-5.26, \mathrm{P}=0.081$ ).

\section{PROs with primary versus secondary prevention ICDs}

As shown in Table 4, patients with secondary prevention ICDs had a lower quality of life score as assessed by LAS when compared to those with primary prevention indications (i.e. $72.0 \pm 23.1$ versus $79.2 \pm 13.0$, relative $\%$ difference $9.01, \mathrm{P}=0.047$ ). There were no significant differences in 
perceived health status, psychological distress, sense of coherence, illness perception, health behaviours, and overall composite score.

\section{Geographic variations in PROs}

Geographic variations in mean differences of the PROs for patients with and without ICDs are portrayed in Figure 2. Panels A and B include PROs for which higher scores indicate better and worse reported outcomes, respectively. On visual inspection, mean differences in PROs, along with their 95\% confidence intervals, tended to cluster below and above the 0 value in Panels A and B, respectively, indicative of worse reported outcomes in patients with ICDs. However, notable regional variations were observed. For example, in the Eastern region, patients with ICDs had significantly worse reported outcomes with respect to satisfaction with life (SWLS), perceived mental health status (SF-12 MCS), psychological distress (HADS), illness perception (Brief IPQ), and health behaviours (HBS-CHD). In Europe and the Americas, ICD recipients reported worse outcomes in physical perceived health status (SF-12 PCS) and illness perception (Brief IPQ). In addition, Europeans with ICDs had a significantly worse sense of coherence (SOC) and those from the Americas had higher anxiety scores (HADS-Anxiety). Finally, ICD recipients from the Pacific region had significantly worse scores for satisfaction with life (SWLS) and health behaviours (HBS-CHD).

Differences in the APPROACH-IS summary score (APPRtot) in patients with and without ICDs are plotted in Figure 3. Marked variability in overall sense of well-being was observed across the various countries. Patients with ICDs in Switzerland, Argentina, USA, and Taiwan had significantly lower summary scores, with non-significantly lower point estimates also observed for the Netherlands and Canada. In contrast, ICD recipients in Belgium had a significantly higher cumulative score. 


\section{DISCUSSION}

To our knowledge, this is the largest study to assess the impact of ICDs on PROs in adults with CHD. Main findings include the following: 1) perceived health status, psychological distress, sense of coherence, and health behaviours are comparable in patients with and without ICDs, and in those with primary versus secondary prevention indications; 2) ICD recipients perceive their illness as more threatening than matched controls without ICDs; 3) the quality of life score (LAS) is significantly lower in those with secondary compared to primary prevention ICD indications; and 4) marked geographic variations are observed in PROs, with a significantly lower overall sense of well-being in ICD recipients in some but not all countries studied.

The psychological impact and adaptive response to an ICD is complex and multifactorial. Psychological distress and concerns regarding ICDs have been reported in approximately $20 \%$ of the general population with ICDs. ${ }^{18}$ Our observation that ICD recipients with CHD perceive their illness as more threatening is, therefore, compatible with these findings. This perception might not be unrealistic given that patients who require an ICD may objectively have a more threatening condition than those who do not. Determinants of a more threatening illness perception, which remain speculative, may include factors such as fear of sudden death and one's ability to cope, social support, intensity of medical follow-up, apprehension about possible shocks, and concerns surrounding the ICD, including complications, malfunction and costs. The non-significant trend towards a higher degree of perceived physical limitations in ICD recipients noted in the current study could potentially contribute to a less favourable illness perception. It remains to be determined whether targeted psychoeducational interventions ${ }^{19}$ could improve psychological outcomes following ICD implantation in adults with CHD.

Among the factors that influence the psychological response of individuals to an ICD are the circumstances surrounding the implant. ${ }^{20}$ Our findings show that adults with CHD who had a 
resuscitated cardiac arrest or sustained ventricular arrhythmia have a significantly worse quality of life score compared to matched patients with primary prevention indications. Individuals with an ICD implanted for a near miss sudden death may have a lower perceived level of control, ${ }^{21}$ be at greater risk for post-traumatic stress disorder following a cardiac arrest ${ }^{22}$ and experience a higher rate of appropriate shocks during follow-up. ${ }^{23}$ Our study could not confirm whether these factors influenced the lower quality of life score in patients with secondary versus primary prevention indications. The difference in point estimates for anxiety scores (HADS-Anxiety), albeit higher in patients with secondary prevention indications (6.9 \pm 4.0 versus $6.2 \pm 3.5)$, did not reach statistical significance. Moreover, information on ICD shocks was not collected in APPROACH-IS. In a prior study of 180 adults with CHD and ICDs, a high degree of shockrelated anxiety was observed, with fears related to consequences of shocks (e.g., creating a scene) and potential triggers (e.g., sexual activity and exercise). ${ }^{24}$

The marked geographic variations observed in the current analysis are compatible with results reported in adults with CHD at large. ${ }^{16}$ Indeed, standard of living and healthcare system factors were previously found to impact PROs above and beyond clinical factors. More specifically, patients from countries with a higher standard of living had a higher composite APPROACH-IS score, with fewer symptoms of depression, and less risky health behaviours. ${ }^{16}$ Such geographic variations may contribute, in part, to inconsistencies in the general literature regarding the impact of ICDs on PROs. ${ }^{25}$ In the current analysis, the ICD population in Eastern countries had worse PROs compared to those without an ICD for all outcomes except physical perceived health status (PCS). Interestingly, ICD recipients in North and South America and Eastern countries but not Europe had significantly higher anxiety scores compared to those without ICDs. However, such subgroup analyses should be considered hypothesis-generating and 
be interpreted with caution in the context of limited sample sizes with multiple testing that may yield spurious associations.

\section{Limitations}

This exploratory study is cross-sectional in nature and subject to associated limitations, including the inability to infer causality. Although every effort was made to produce balanced groups with regards to characteristics through propensity score weight-matching, the analyses could not adjust for unknown or unmeasured potential confounders. The APPROACH-IS study collected comprehensive information regarding ICD implantation dates and indications. However, procedure-related complications and shocks were not assessed. These potential explanatory variables could have shed further light on the impact of ICDs on PROs. Most subjects were recruited in clinic, which may result in a referral bias, as reflected by the low proportion of enrolled patients with simple forms of CHD. While this issue has implications regarding generalizability, it should not impact internal validity since the groups compared were wellbalanced with respect to CHD complexity. The response rate and characteristics of patients who did not return questionnaires were unknown for most countries. Systematic differences in PROs between responders and non-responders could potentially impact PRO scores. Nevertheless, if such a measurement error is present, it is most likely non-differential and not a threat to the validity of two-group comparisons. Furthermore, patients from all continents except Africa were included. Future studies are required to assess the impact of ICDs on PROs in countries not represented. 


\section{CONCLUSION}

In conclusion, in a large international study of PROs in adults with CHD, perceived health status, psychological distress, sense of coherence, and health behaviours were comparable in those with and without ICDs. However, ICDs were associated with a more threatening perception of illness. Patients with ICDs implanted for secondary prevention reported a worse quality of life score than their counterparts with primary prevention indications despite adjusting for complexity of CHD, heart failure, and other comorbidities. Importantly, marked geographic variations in PROs were observed, reflecting the importance of cultural and socioeconomic factors on reported outcomes. These findings call attention to the need to address psychological concerns in ICD recipients with CHD and pave the way for future studies to assess targeted interventions.

\section{ACKNOWLEDGEMENTS}

None 


\section{FIGURE LEGENDS}

\section{Figure 1. LOVE plot}

A LOVE plot depicts the percentage of pooled weighted standard deviations for the standard differences in weighted means or weighted proportions for each covariate across exposure groups before $(\mathrm{X})$ and after $(\mathrm{O})$ matching. The dotted blue and red lines represent $10 \%$ and $5 \%$ cut-off values, respectively.

CHF denotes congestive heart failure; CHD, congenital heart disease

\section{Figure 2. Regional variations in PROs}

Differences in estimated means of the various PROs between patients with and without ICDs are plotted according to geographical region (Eastern, Europe, North and South America, and Pacific). Panel A includes PROs for which a higher score indicates a better outcome, whereas Panel B captures PROs for which a lower score reflects a worse outcome. See Table 1 for abbreviations of the various PROs.

\section{Figure 3. Difference in summary score for PROs in patients with and without ICDs according to country}

Differences in cumulative APPRtot scores between patients with and without ICDs are plotted according to country and are color-coded by region. Participating countries with at least three ICD recipients are included in the graph. 


\section{TABLES}

\section{Table 1. Summary of patient-reported outcomes (PROs)}

\begin{tabular}{|c|c|c|}
\hline Questionnaire & Scale & Description \\
\hline \multicolumn{3}{|l|}{ Quality of life } \\
\hline Linear analogue scale (LAS) & $0-100$ & $\begin{array}{l}\text { Vertical line: higher score reflects better quality of } \\
\text { life }\end{array}$ \\
\hline Satisfaction with life scale (SWLS) & $5-35$ & $\begin{array}{l}5 \text { statements with scores from } 1 \text { to } 7 \text {; higher score } \\
\text { reflects better quality of life }\end{array}$ \\
\hline \multicolumn{3}{|l|}{ Perceived health status } \\
\hline $\begin{array}{l}\text { 12-item Short-Form Health Survey } \\
\text { version } 2 \text { (SF-12); divided into physical } \\
\text { (PCS) and mental (MCS) component } \\
\text { scores }\end{array}$ & $\begin{array}{l}0-100 \text { for both PCS } \\
\text { and MCS }\end{array}$ & $\begin{array}{l}\text { Higher score reflects better perceived health status } \\
8 \text { health domains: } \\
\text { - PCS: 1) Physical functioning; 2) Role } \\
\text { participation with physical health problems; } \\
\text { 3) Bodily pain; 4) General health } \\
\text { MCS: 5) Vitality; 6) Social functioning; } \\
\text { 7) Emotional health; 8) Mental health }\end{array}$ \\
\hline $\begin{array}{l}\text { European Quality of Life - } 5 \\
\text { Dimension Score (EQ-5D) }\end{array}$ & $5-15$ & $\begin{array}{l}\text { Higher score reflects lower perceived health status } \\
\text { - } 5 \text { dimensions: 1) Mobility; 2) Self-care; } \\
\text { 3) Usual activities; 4) Pain and discomfort; } \\
\text { 5) Anxiety and depression }\end{array}$ \\
\hline \multicolumn{3}{|l|}{ Psychological distress } \\
\hline $\begin{array}{l}\text { Hospital Anxiety (HADS-Anxiety) and } \\
\text { Depression (HADS-Depression) Scale }\end{array}$ & $\begin{array}{l}0-21 \text { for depression } \\
\text { and anxiety }\end{array}$ & Higher score reflects greater psychological distress \\
\hline \multicolumn{3}{|l|}{ Sense of coherence } \\
\hline $\begin{array}{l}\text { Sense of coherence score - orientation } \\
\text { to life questionnaire (SOC) }\end{array}$ & $13-91$ & $\begin{array}{l}\text { Higher score reflects higher sense of coherence } \\
\text { - } 3 \text { components: 1) Comprehensibility; } \\
\text { 2) Manageability; 3) Meaningfulness }\end{array}$ \\
\hline \multicolumn{3}{|l|}{ Illness perception } \\
\hline $\begin{array}{l}\text { Brief illness perception questionnaire } \\
\text { (Brief IPQ) }\end{array}$ & $0-80$ & $\begin{array}{l}\text { Higher score reflects worse illness perception } \\
\text { 9 items: 1) Consequences; 2) Timeline; } \\
\text { 3) Personal control; 4) Treatment control; } \\
\text { 5) Identity; 6) Coherence; 7) Concern; } \\
\text { 8) Emotional response; 9) Perceived causes }\end{array}$ \\
\hline \multicolumn{3}{|l|}{ Health behaviors } \\
\hline $\begin{array}{l}\text { Health-Behavior Scale-Congenital } \\
\text { Heart Disease (HBS-CHD) }\end{array}$ & $0-7$ & $\begin{array}{l}\text { Higher score reflects greater health risk } \\
\text { - } 3 \text { items: 1) Substance abuse; 2) Physical } \\
\text { activity; 3) Dental hygiene }\end{array}$ \\
\hline \multicolumn{3}{|l|}{ Overall well-being } \\
\hline APPROACH-IS total score (APPRtot) & $0-100$ & $\begin{array}{l}\text { Higher score reflects better state of well-being } \\
\text { Composite of all PROs listed above }\end{array}$ \\
\hline
\end{tabular}


Table 2. Characteristics in matched cohorts with and without ICDs

\begin{tabular}{|c|c|c|c|}
\hline & $\begin{array}{c}\text { ICD } \\
\mathbf{N}=107\end{array}$ & $\begin{array}{l}\text { No ICD } \\
\mathrm{N}=3,081\end{array}$ & P-value \\
\hline Age (years) & $40.1 \pm 12.4$ & $40.2 \pm 14.1$ & 0.462 \\
\hline Female sex $(\%)$ & 50.5 & 50.4 & 0.985 \\
\hline Ethnicity (\%) & & & 1.000 \\
\hline Middle-Eastern/Arabic & 0.9 & 0.9 & \\
\hline Asian & 5.6 & 5.5 & \\
\hline African & 0.0 & 0.0 & \\
\hline Hispanic & 0.9 & 0.9 & \\
\hline White & 92.5 & 92.6 & \\
\hline Educational level (\%) & & & 0.919 \\
\hline Less than high school & 4.7 & 4.6 & \\
\hline High school & 42.1 & 42.4 & \\
\hline College & 29.9 & 30.6 & \\
\hline University & 23.4 & 23.4 & \\
\hline Marital status (\%) & & & 0.987 \\
\hline Unmarried/never married & 35.5 & 36.0 & \\
\hline Separated, divorced or widowed & 5.6 & 5.8 & \\
\hline Married or with partner & 58.9 & 58.2 & \\
\hline Tobacco use (\%) & 8.4 & 8.6 & 0.829 \\
\hline Cognitive impairment (\%) & 1.9 & 1.7 & 0.686 \\
\hline Complexity of congenital heart disease (\%) & & & 0.381 \\
\hline Simple & 4.7 & 4.5 & \\
\hline Moderate & 54.2 & 52.0 & \\
\hline Complex & 41.1 & 43.6 & \\
\hline Congestive heart failure (CHF) (\%) & & & 0.826 \\
\hline No history of CHF & 58.9 & 58.0 & \\
\hline Past history of $\mathrm{CHF}$ & 18.7 & 18.7 & \\
\hline Current CHF & 22.4 & 23.3 & \\
\hline
\end{tabular}


Table 3. Characteristics in matched cohorts with primary and secondary prevention ICDs

\begin{tabular}{|c|c|c|c|}
\hline & $\begin{array}{c}\text { Primary } \\
\text { prevention } \\
\mathrm{N}=41\end{array}$ & $\begin{array}{l}\text { Secondary } \\
\text { prevention } \\
\mathbf{N}=\mathbf{6 3}\end{array}$ & P-value \\
\hline Age (years) & $40.0 \pm 12.1$ & $40.2 \pm 12.6$ & 0.929 \\
\hline Female sex $(\%)$ & 55.1 & 54.7 & 0.964 \\
\hline Time since ICD implantation (years) & $7.3 \pm 8.1$ & $7.6 \pm 7.1$ & 0.852 \\
\hline Ethnicity (\%) & & & 0.999 \\
\hline Middle-Eastern/Arabic & 0.0 & 0.0 & \\
\hline Asian & 5.9 & 5.5 & \\
\hline African & 0.0 & 0.0 & \\
\hline Hispanic & 0.0 & 0.0 & \\
\hline White & 94.1 & 94.5 & \\
\hline Educational level (\%) & & & 0.991 \\
\hline Less than high school & 4.0 & 5.1 & \\
\hline High school & 46.9 & 44.6 & \\
\hline College & 26.3 & 26.6 & \\
\hline University & 22.8 & 23.8 & \\
\hline Marital status (\%) & & & 0.999 \\
\hline Unmarried/never married & 35.5 & 35.8 & \\
\hline Separated, divorced or widowed & 5.9 & 5.8 & \\
\hline Married or with partner & 58.6 & 58.4 & \\
\hline Tobacco use $(\%)$ & 5.9 & 5.6 & 0.939 \\
\hline Cognitive impairment $(\%)$ & 1.3 & 1.3 & 0.998 \\
\hline Complexity of congenital heart disease (\%) & & & 0.967 \\
\hline Simple & 3.3 & 4.3 & \\
\hline Moderate & 51.7 & 51.2 & \\
\hline Complex & 45.0 & 44.5 & \\
\hline Congestive heart failure (CHF) (\%) & & & 0.995 \\
\hline No history of CHF & 59.2 & 56.6 & \\
\hline Past history of CHF & 14.8 & 16.7 & \\
\hline Current $\mathrm{CHF}$ & 26.1 & 26.7 & \\
\hline
\end{tabular}


Table 4. Comparisons of PROs in matched cohorts with an ICD versus no ICD and in those with primary versus secondary prevention indications

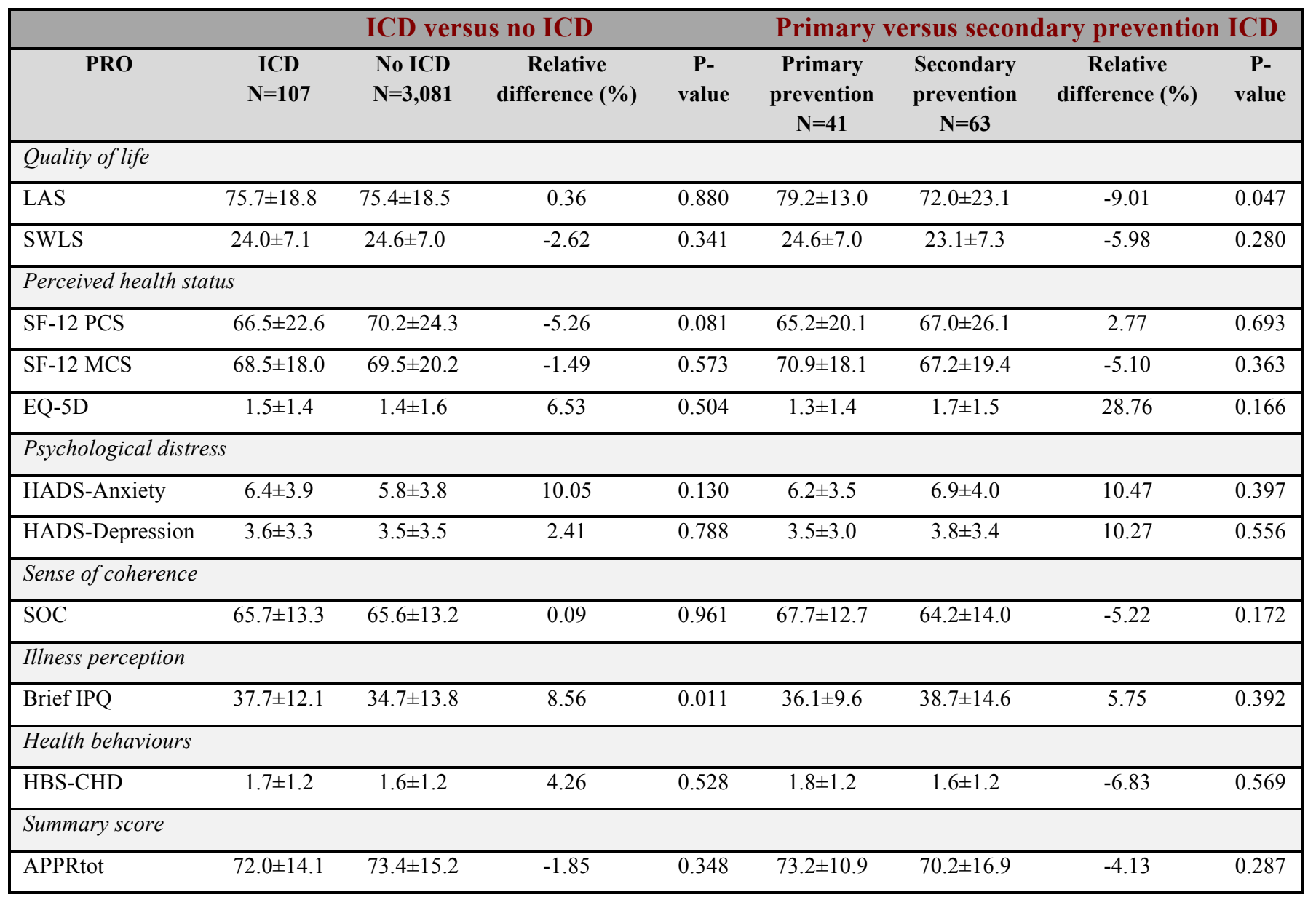




\section{REFERENCES}

1. Khairy P, Van Hare GF, Balaji S, et al. PACES/HRS Expert consensus statement on the recognition and management of arrhythmias in adult congenital heart disease. Heart Rhythm 2014;11:e102-e165.

2. Thylen I, Dekker RL, Jaarsma T, Stromberg A, Moser DK. Characteristics associated with anxiety, depressive symptoms, and quality-of-life in a large cohort of implantable cardioverter defibrillator recipients. J Psychosom Res 2014;77:122-127.

3. Ferguson $\mathrm{M}$ and Kovacs AH. An integrated adult congenital heart disease psychology service. Congenit Heart Dis. 2016;11:444-451.

4. Stout KK, Daniels CJ, Aboulhosn JA, et al. 2018 AHA/ACC guideline for the management of adults with congenital heart disease. J Am Coll Cardiol doi: 10.1016/j.jacc.2018.08.1029.

5. Apers S, Kovacs AH, Luyckx K, et al. Assessment of Patterns of Patient-Reported Outcomes in Adults with Congenital Heart disease - International Study (APPROACH-IS): rationale, design, and methods. Int J Cardiol. 2015;179:334-342.

6. Warnes CA, Williams RG, Bashore TM, et al. ACC/AHA 2008 Guidelines for the management of adults with congenital heart disease. Circulation 2008;118:e714-e833.

7. Moons P, Budts W, De Geest S. Critique on the conceptualisation of quality of life: a review and evaluation of different conceptual approaches. Int J Nurs Stud 2006;43:891-901.

8. Diener E, Emmons RA, Larsen RJ, Griffin S. The Satisfaction With Life Scale. J Pers Assess 1985;49:71-75.

9. Rumsfeld JS. Health status and clinical practice: when will they meet? Circulation 2002;106:5-7.

10. Johnson JA, Coons SJ. Comparison of the EQ-5D and SF-12 in an adult US sample. Qual Life Res 1998;7:155-166.

11. Zigmond AS, Snaith RP. The hospital anxiety and depression scale. Acta Psychiatr Scand 1983;67:361-370.

12. Muller J, Hess J, Hager A. Sense of coherence, rather than exercise capacity, is the stronger predictor to obtain health-related quality of life in adults with congenital heart disease. Eur J Prev Cardiol 2014;21:949-955.

13. Antonovsky A. The structure and properties of the sense of coherence scale. Soc Sci Med. 1993;36:725-733.

14. Broadbent E, Petrie KJ, Main J, Weinman J. The brief illness perception questionnaire. J Psychosom Res 2006;60:631-637.

15. Goossens E, Luyckx K, Mommen N, et al. Health risk behaviors in adolescents and emerging adults with congenital heart disease: psychometric properties of the Health Behavior Scale-Congenital Heart Disease. Eur J Cardiovasc Nurs 2013;12:544-657.

16. Moons P, Kovacs AH, Luyckx K, et al. Patient-reported outcomes in adults with congenital heart disease: Inter-country variation, standard of living and healthcare system factors. Int $\mathrm{J}$ Cardiol 2018;251:34-41.

17. Li L, Greene T. A weighting analogue to pair matching in propensity score analysis. Int $J$ Biostat 2013;9:215-234.

18. Magyar-Russell G, Thombs BD, Cai JX, et al. The prevalence of anxiety and depression in adults with implantable cardioverter defibrillators: a systematic review. J Psychosom Res 2011;71:223-231. 
19. Kao CW, Chen MY, Chen TY, Lin PH. Effect of psycho-educational interventions on quality of life in patients with implantable cardioverter defibrillators: a meta-analysis of randomized controlled trials. Health Qual Life Outcomes 2016;14:138.

20. Sears SF, Hauf JD, Kirian K, Hazelton G, Conti JB. Posttraumatic stress and the implantable cardioverter-defibrillator patient: what the electrophysiologist needs to know. Circ Arrhythm Electrophysiol 2011;4:242-250.

21. Hammash M, McEvedy SM, Wright J, et al. Perceived control and quality of life among recipients of implantable cardioverter defibrillator. Aust Crit Care 2018. doi: 10.1016/j.aucc.2018.08.005.

22. von Kanel R, Baumert J, Kolb C, Cho EY, Ladwig KH. Chronic posttraumatic stress and its predictors in patients living with an implantable cardioverter defibrillator. J Affect Disord 2011;131:344-352.

23. Sweeney MO, Wathen MS, Volosin K, et al. Appropriate and inappropriate ventricular therapies, quality of life, and mortality among primary and secondary prevention implantable cardioverter defibrillator patients. Circulation 2005;111:2898-905.

24. Cook SC, Valente AM, Maul TM, et al. Shock-related anxiety and sexual function in adults with congenital heart disease and implantable cardioverter defibrillators. Heart Rhythm 2013;10:805-810.

25. Ooi SL, He HG, Dong Y, Wang W. Perceptions and experiences of patients living with implantable cardioverter defibrillators: a systematic review and meta-synthesis. Health Qual Life Outcomes 2016;14:160. 


\section{FIGURES}

Figure 1

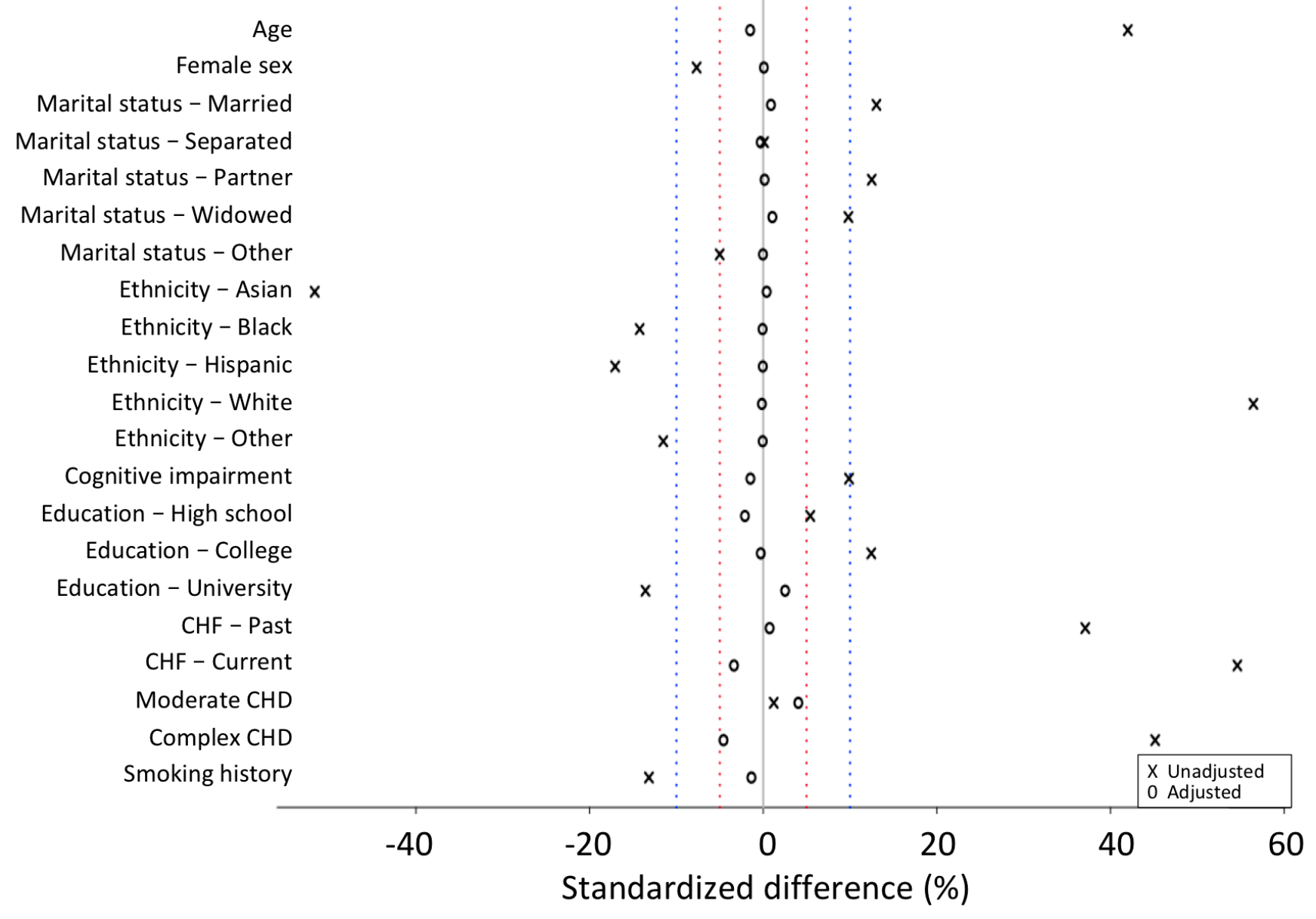


Figure 2

A

PROs with higher scores indicative of better outcomes

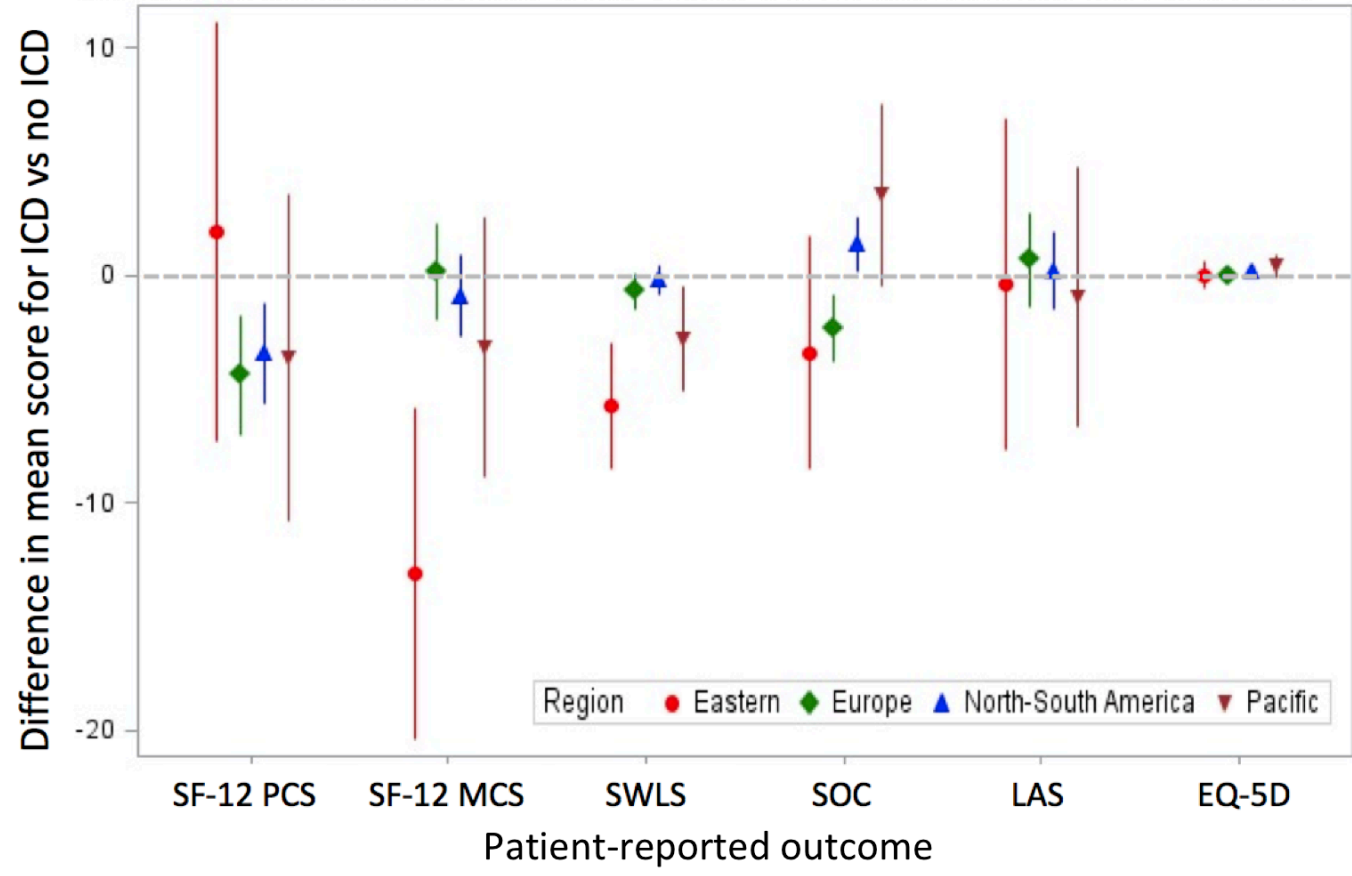

B

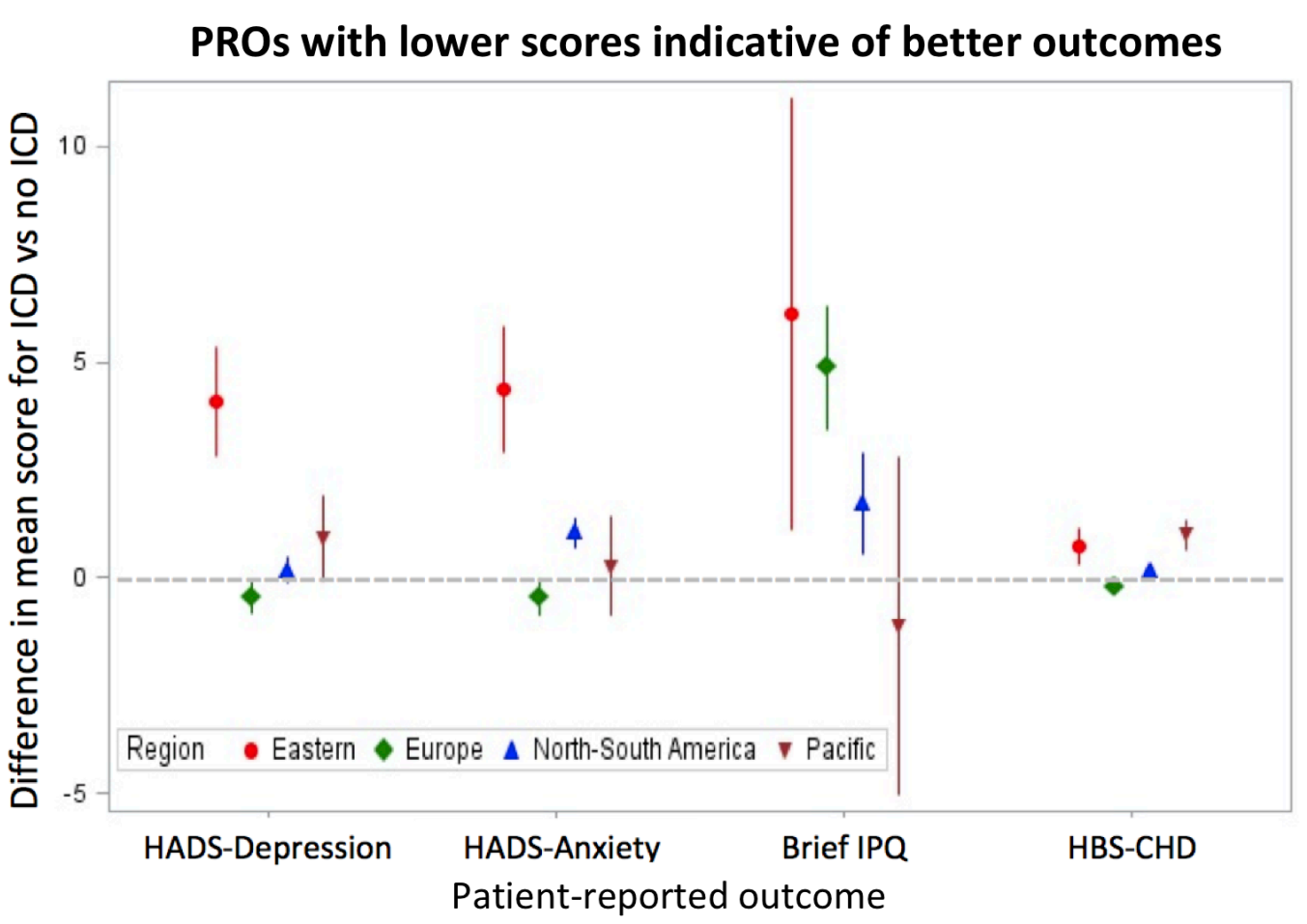


Figure 3

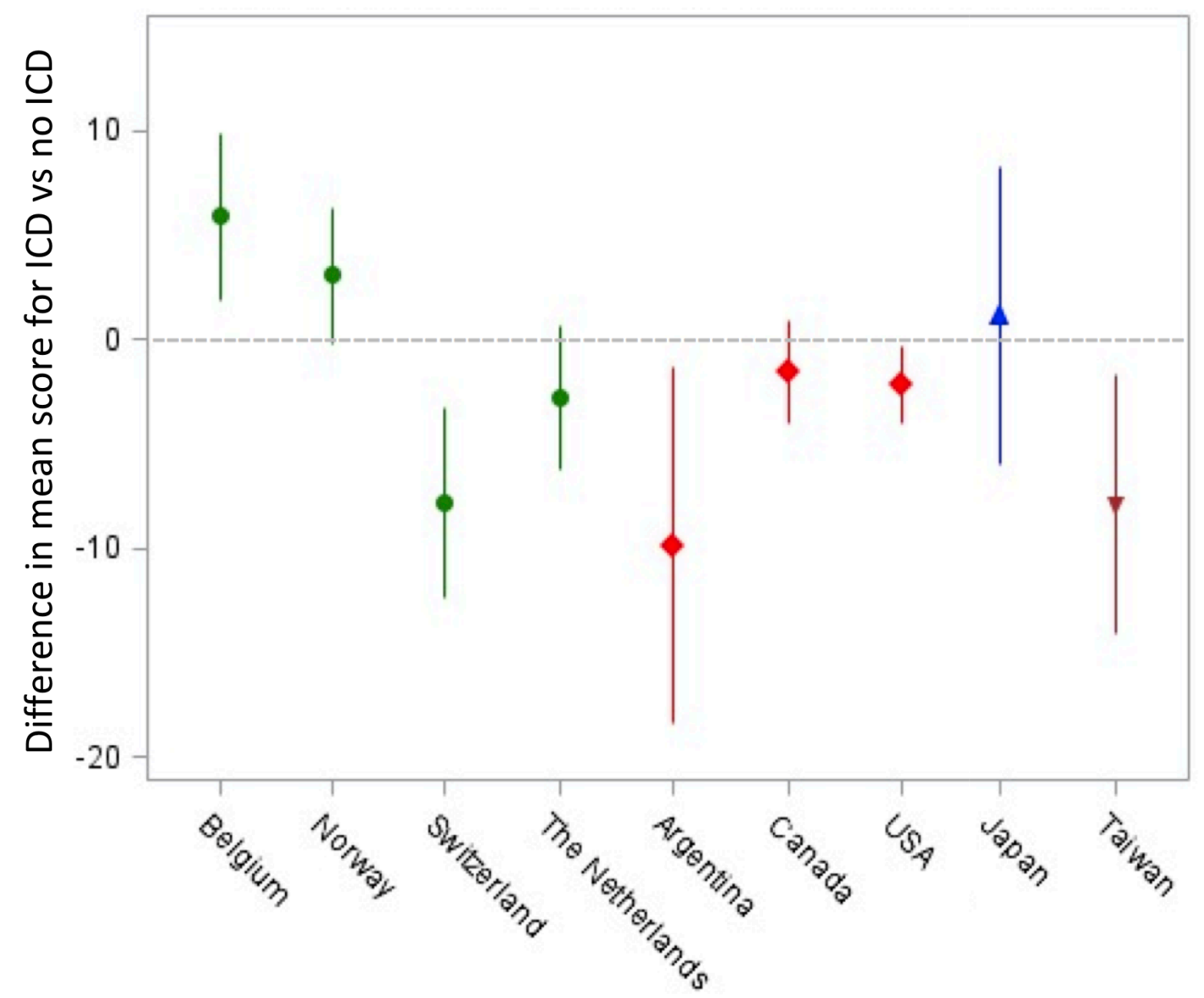

Countries

Region - Europe $\bullet$ North-South America $\Delta$ Pacific $\mathbf{v}$ Eastern 